\title{
Spin-caloric transport properties of cobalt nanostructures: spin disorder effects from first principles
}

\author{
Roman Kováčik * Phivos Mavropoulos, Daniel Wortmann, and Stefan Blügel \\ Peter Grünberg Institut and Institute for Advanced Simulation, \\ Forschungszentrum Jülich and JARA, 52425 Jülich, Germany
}

(Dated: July 3, 2021)

\begin{abstract}
The fundamental aspects of spin-dependent transport processes and their interplay with temperature gradients, as given by the spin Seebeck coefficient, are still largely unexplored and a multitude of contributing factors must be considered. We used density functional theory together with a Monte-Carlo-based statistical method to simulate simple nanostructures, such as Co nanowires and films embedded in a $\mathrm{Cu}$ host or in vacuum, and investigated the influence of spin-disorder scattering on electron transport at elevated temperatures. While we show that the spin-dependent scattering of electrons due to temperature induced disorder of the local magnetic moments contributes significantly to the resistance, thermoelectric and spin-caloric transport coefficients, we also conclude that the actual magnitude of these effects cannot be predicted, quantitatively or qualitatively, without such detailed calculations.

PACS numbers: 75.76.+j, 72.15.Jf, 72.10.-d
\end{abstract}

\section{INTRODUCTION}

The recent discovery of the spin Seebeck effect $\underline{\underline{1}}$ triggered a broad discussion about its microscopic origin and correct interpretation of the measured data (spin Seebeck versus spin-dependent Seebeck effect), $\underline{\underline{2}} \underline{\underline{10}}$ and extended the field of spin-caloritronics $\stackrel{11}{\Perp}$ which investigates the coupling between electrical, spin, and heat transport. Possible technological importance was suggested for the spin Seebeck effect in thermally-driven position sensing $\stackrel{12.13}{\underline{1}}$ Furthermore, an enhanced Peltier effect reported in submicron-sized metallic junctions could lead to applications in electronics micro-cooling $\underline{14}$ 17 Ferromagnetic materials subject to a temperature gradient experience, in addition to a heat current (thermal conductivity) and a charge current (thermoelectric effect), an induced spin current (spin-caloric effect). In metals we expect a large part of the spin current to arise from spinpolarized electron propagation due to generally different conductivity for the majority and minority spin channels, while other possible effects, e.g., spin transport due to magnons or even phonons, are dominant in insulators.

Theoretical and computational investigation is an essential part of understanding spin-caloric phenomena due to a non-trivial connection between the microscopic character of relevant materials and their functionality. The asymmetry of the electronic transmission coefficient as a function of energy around the Fermi level $\left(E_{\mathrm{F}}\right)$ enters the expressions for the thermoelectric effects, making quantitative or even semi-quantitative predictions next to impossible on a simple model level. Given the complexity of the electronic structure, numerical calculations are therefore inevitable.

An important contribution to the electron-transport phenomena at high temperatures is the formation of a spin-disordered state due to local-moment fluctuations in the magnetic material. This comes on top of the phonon contribution at high temperatures and the spin- orbit contribution to spin mixing that occurs at all temperatures $\underline{\underline{18}}$ The fluctuations induce spin-conserving and spin-flip scattering and clearly contribute to the temperature-dependent transport phenomena. For example, they induce the well-known spin disorder resistivity that has been experimentally investigated in the past in ferromagnetic materials,,$\frac{19,20}{1}$ and was successfully modeled with ab-initio techniques $\stackrel{21-25}{=}$ It is obvious that spin fluctuations must also contribute to thermoelectric and spin-caloric phenomena. However, this effect has not been investigated so far. In the present paper we address this problematics by means of density-functional calculations and Monte-Carlo simulations and arrive at the conclusion that the impact of temperature-induced spin disorder is strong at temperatures where the magnetization reduction is significant. We also find that there is no universal correlation between the temperature-dependent magnetization and the thermoelectric and spin-caloric coupling, i.e., it has to be examined separately for each material and microscopic structure and at each temperature, due to the delicate modulation of the electronscattering as a function of energy around $E_{\mathrm{F}}$ and as a function of temperature.

We focus on magnetic nanostructures, particularly on Co nanocolumns embedded between $\mathrm{Cu}$ leads, motivated by the miniaturization of spintronics devices and by recent suggestions that nanostructured magnetic materials (e.g., in the "Konbu" phase) can lead to extraordinary thermoelectric effects due to quantum confinement $\underline{\underline{16,17}}$ In addition, we approach the bulk limit by considering the transport through a thin layer formed by several atomic layers of Co. The electronic structure of the studied systems is calculated within the multiple scattering screened Korringa-Kohn-Rostoker Green function (KKRGF) framework using the full-potential formalism. ${ }^{26}$ The Monte-Carlo methodology is then used to simulate the effect of temperature on the magnetic configurations within a Heisenberg model with the exchange coupling 
parameters calculated according to Liechtenstein et al $\stackrel{27}{\stackrel{27}{r}}$ The transmission probability through the spin-disordered magnetic structures is obtained using the LandauerBüttiker approach for the ballistic transport within the

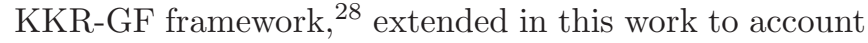
for the non-collinear magnetic effects similar to work in Ref. 29, thus providing individual spin-preserving and spin-flip contributions to the transmission probability. The electrical conductance and Seebeck coefficients are finally calculated from the transport coefficients.

In the following, we describe our model systems and briefly summarize methodologies used in this work and corresponding computational details (Sec. III). Results are presented and discussed in Sec. III and our main conclusions are given in Sec. IV]

\section{METHODOLOGY}

\section{A. Geometric structure}

The geometry that we choose is intended to serve as a generic model of Co nanostructures embedded, on the one hand, between free-electron-metal leads, and on the other hand surrounded in the lateral direction either by a free-electron metal or by vacuum or an insulating material modeled here by vacuum. We choose a few different geometries to see if the effect of spin-disorder on the transport coefficients in the nano-scale is significantly affected by the magnetic nanostructure shape and size and by the surrounding medium (metallic or insulating).

The basic setup of our model systems is depicted in Fig. 1 The left and right semi-infinite leads consist of the fcc crystal lattice of $\mathrm{Cu}$ atoms with the experimental lattice constant $a_{\text {lat }}=3.62 \AA$ and with the interface to the scattering region orthogonal to the $z$ axis ([001] direction). The region between the leads contains 8 atomic layers of either Co atoms forming a thin layer or Co atoms in a shape of a thin wire in various structural configurations surrounded by $\mathrm{Cu}$ or vacuum (see Fig. (2). The sites in the scattering region epitaxially follow the perfect fcc lattice of the leads, which is an acceptable approximation due to the small size of this region. Since our focus is to study the effect of spin disorder, the atoms are kept at their ideal unrelaxed positions. A supercell approach with two-dimensional periodicity within the $x y$ plane is used to model the real space spin disorder in a thin layer of Co atoms [Fig. 2(c)], as well as for all wire-like Co structures [Fig. 2(a-b,d-f)], imposing a separation of at least $2 a_{\text {lat }}$ to any atom in their periodic images. The transmission probability between the left and right lead is evaluated for pairs of $\mathrm{Cu}$ atomic layers placed sufficiently away from the interface with the magnetic region so that the $\mathrm{Cu}$ potentials are bulk-like.

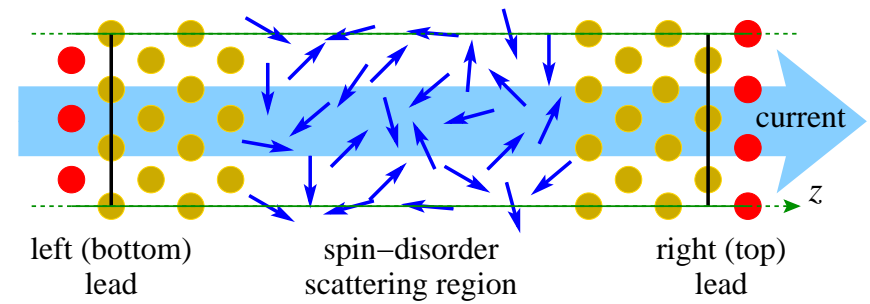

FIG. 1: (Color online) General setup of our model system with atoms placed on the fcc crystal lattice. The direction of current is parallel with the $z$ axis ([001] direction). $\mathrm{Cu}$ and Co atoms are shown as spheres and arrows, respectively. The transmission probability is evaluated for pairs of atomic layers (indicated by vertical lines) placed far enough from the $\mathrm{Cu} / \mathrm{Co}$ interface.

\section{B. Electronic structure}

The KKR-GF method using the full-potential formalism ${ }^{26.30}-32$ and local density approximation 33 to the exchange-correlation energy functional is employed to calculate the electronic structure of our model systems, for which the angular moment expansion is truncated after $l_{\max }=3$. We calculate the electronic structure of the ground state in a multiple step procedure.

In the first step we calculate the self-consistent density and potential of a "slab" system $\mathrm{Cu}(7)-\{\mathrm{Cu} / \mathrm{Co} / \mathrm{Va}\}(8)-$ $\mathrm{Cu}(7)$ with one atom per layer. Here, the "scattering region" $\{\mathrm{Cu} / \mathrm{Co} / \mathrm{Va}\}(8)$ consists of eight layers of $\mathrm{Co}$ atoms, $\mathrm{Cu}$ atoms or vacuum, depending on the system. This region is sandwiched between two $\mathrm{Cu}(7)$ regions, consisting of seven atomic layers of $\mathrm{Cu}$, and the whole slab is embedded in vacuum. A well converged density was reached using a $36 \times 36 \mathrm{k}$-point mesh for the integration in the surface Brillouin zone (SBZ) and a smearing temperature of $800 \mathrm{~K}$.

In a second step, we replace the outermost part of the slab, i.e., the three outermost $\mathrm{Cu}$ atomic layers and the outer vacuum, by half-infinite $\mathrm{Cu}$ leads. Then, employing the decimation technique, $\stackrel{34,35}{=}$ we use the central potential of the $\mathrm{Cu}(7)$ layer that is already bulk-like to a very good approximation to construct the self-energy induced by the half-infinite region. In other words, we attach the half-infinite leads $(\cdots \mathrm{Cu}$ - and $-\mathrm{Cu} \cdots)$ on the $\mathrm{Cu}(4)$ $\{\mathrm{Cu} / \mathrm{Co} / \mathrm{Va}\}(8)-\mathrm{Cu}(4)$ "central" part of the slab.

The third and final step corresponds to the construction of supercell potentials. At this point, the $\cdots \mathrm{Cu}-$ $\mathrm{Cu}(4)-\mathrm{Co}(8)-\mathrm{Cu}(4)-\mathrm{Cu} \cdots$ system forms a base for the model system of the thin Co layer (TL). A $3 \times 3$ supercell [shown in Fig. 2(c)] is constructed by replicating the individual site potentials. To obtain the electronic structure of all other systems (nanowires), the embedded Co wire and the nearest neighbors were further treated self-consistently by the impurity Green function method, 26,37 Here, we use as a reference the $\mathrm{Cu}(4)$ $\mathrm{Cu}(8)-\mathrm{Cu}(4)$ system (Cu bulk) for the nanowires embedded in $\mathrm{Cu}[$ Fig. 2(a,b)] or the $\mathrm{Cu}(4)-\mathrm{Va}(8)-\mathrm{Cu}(4)$ system 


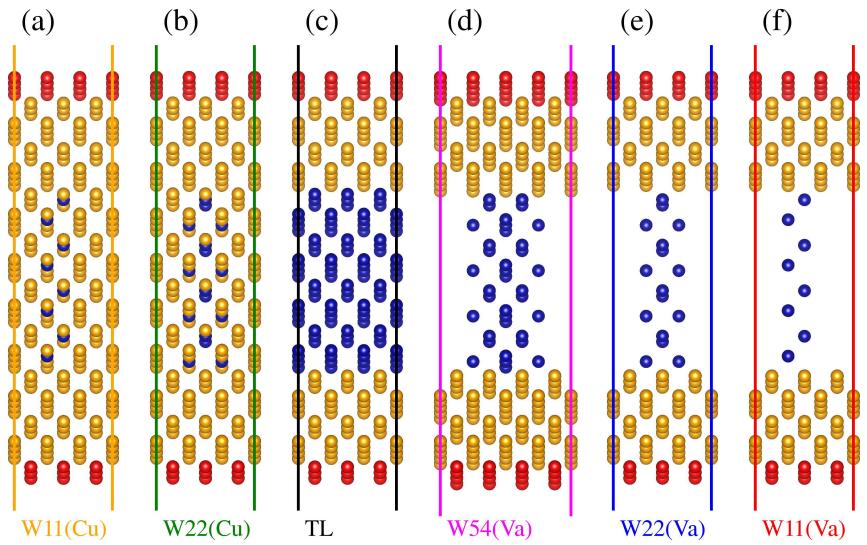

FIG. 2: (Color online) Cobalt (blue/dark spheres) nanostructures sandwiched between $\mathrm{Cu}$ leads (yellow and red/bright spheres) used in this study: (c) thin Co layer "TL" consisting of 8 monolayers, $(\mathrm{a}, \mathrm{f})$ monoatomic wires "W11(Cu)" and "W11(Va)" having 1 Co atom in all layers, (b,e) biatomic wires "W22(Cu)" and "W22(Va)" having 2 Co atoms in all layers, (d) wire "W54(Va)" with alternating 5 and 4 $\mathrm{Co}$ atoms. The $(\mathrm{Cu})$ and $(\mathrm{Va})$ indicate the type of embedding (copper and vacuum, respectively) of the Co nanostructure. Periodic boundaries are indicated by solid lines. Crystal structures were plotted using VESTA ${ }^{36}$

for the nanowires embedded in vacuum [Fig. 2(d,e,f)]. The structure of the nanowires is shown from a side view in Fig. 2(a-b,d-f) and from a top view in Fig. 3. Inclusion of the second nearest neighbors in the impurity cluster led to negligible differences of occupation $(<0.006$ electron $)$ and magnetic moments $\left(<0.005 \mu_{\mathrm{B}}\right)$ of the cobalt atoms and their nearest neighbor sites. The occupation of second nearest neighbors did not differ more than 0.011 electron from the unperturbed reference site in their respective layer. Finally, a supercell in the $x y$ plane, i.e., perpendicular to the lead/wire interface was formed by the converged potentials of the impurity cluster sites embedded in the respective reference potentials of $\{\mathrm{Cu} / \mathrm{Va}\}$. The nanowires $\mathrm{W} 11(\mathrm{Cu} / \mathrm{Va})$ and $\mathrm{W} 22(\mathrm{Cu} / \mathrm{Va})$ were modeled in the $3 \times 3$ supercell, whereas the W54(Va) wire, having a larger cross-section, was modeled in the $4 \times 4$ supercell (see also Fig. 2).

\section{Spin disorder}

Our model of the spin disorder is based on adopting the moment directions as they are given by a classical Heisenberg model at non-zero temperature. In this spirit we set up a Heisenberg Hamiltonian

$$
H=-\sum_{i j} J_{i j} \mathbf{M}_{i} \cdot \mathbf{M}_{j}
$$

where $\mathbf{M}_{i}$ and $\mathbf{M}_{j}$ are unit vectors pointing in the direction of the moments at sites $i$ and $j$, respectively, while $J_{i j}$ are the exchange parameters extracted from the (a)

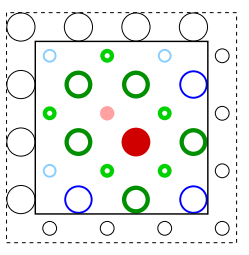

(b)

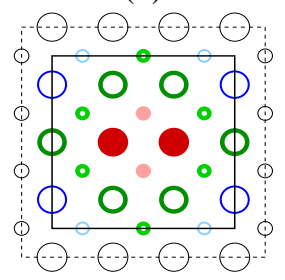

(c)

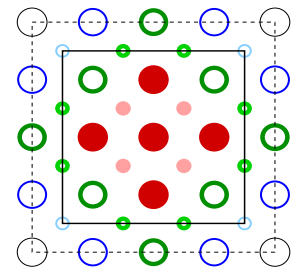

FIG. 3: (Color online) Setup of the nanowires geometry for the (a) W11, (b) W22 and (c) W54 nanowires: Co sites (red / filled circle), their first nearest neighbor (green / thick line circle), second nearest neighbor (blue / medium thick line circle) and embedding sites (black / thin line circle) are shown in the two adjacent layers (large and small circles, respectively). The $3 \times 3$ and $4 \times 4$ supercell is indicated by solid and dashed line, respectively.

ground-state electronic structure. Based on the formal-

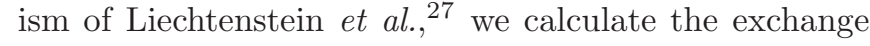
coupling parameters $J_{i j}$ between the Co atoms.

The thermal fluctuations of the magnetic moments of Co atoms are modeled by the Monte-Carlo (MC) approach using the Metropolis algorithm $\frac{38}{2}$ As a random number generator we used the Mersenne twister $\stackrel{39}{\underline{3}}$ Since the statistics of quantities evaluated from the spin configurations strongly depends on the temperature and character of a particular system, we empirically determine the number of required MC configurations $N_{\text {conf }}$ by a simple criterion that accounts for the fluctuation amplitude,

$$
N_{\text {conf }}=N_{0} \sqrt{\left\langle m_{\mathrm{MC}}^{2}\right\rangle-\left\langle m_{\mathrm{MC}}\right\rangle^{2}} /\left\langle m_{0}\right\rangle
$$

where $m_{\mathrm{MC}}$ is the magnitude of the system magnetization at a given $\mathrm{MC}$ snapshot, $\left\langle m_{\mathrm{MC}}\right\rangle$ its average over MC configurations, $\left\langle m_{\mathrm{MC}}^{2}\right\rangle$ the MC average of $m_{\mathrm{MC}}^{2}$ and $\left\langle m_{0}\right\rangle$ the magnitude of the ground-state magnetization in the KKR-GF calculation. The empirical constant $N_{0}$ was set to 5000 yielding typical $N_{\text {conf }} \approx 800$ around the crossover temperature where the fluctuations are most pronounced. The spin-up and spin-down directions in the electronic structure derived quantities at each MC snapshot are given with respect to the global magnetization axis of the same MC snapshot and averaged at the end over all snapshots. Besides monitoring the evolution of average magnetic moment and magnetic susceptibility as a function of temperature, we calculate the correlation function $C_{N}$ between moments at different layer pairs, where $N a_{\text {lat }} / 2$ is the distance between the two layers. In the calculation of $C_{N}$ we include correlations between moments at atomic sites whose distance, when projected onto the $x y$ plane, does not exceed $0.5 a_{\text {lat }}$. Using indices $a$ and $b$ for the in-plane position and $c, d$ for the layers, we define

$$
C_{N}(T)=\frac{1}{N_{a} N_{b}} \sum_{a, b} \frac{1}{N_{c d}} \sum_{c<d}\left\langle\mathbf{M}_{a b c} \cdot \mathbf{M}_{a b d}\right\rangle_{T},
$$

where $N_{c d}$ is the number of all layer pairs with distance 
$N a_{\text {lat }} / 2$, and $N_{a}$ and $N_{b}$ is the number of magnetic sites along the $x$ and $y$ directions of the MC supercell.

\section{Electron transport}

In order to evaluate the transmission probability matrix using the formalism of non-collinear magnetism, a code was developed interfacing the existing KKR-GF 26 and transport $\underline{28}$ programs, which were modified accordingly to treat the non-collinear magnetic states. We make implicit use of the adiabatic approximation, assuming that the electron traverses the nanostructure or junction at a faster timescale compared to the precession of localized moments $\stackrel{40,41}{4}$ Our approach amounts to a rotation of the ground-state magnetic part of the site-dependent potentials in the direction prescribed by the MC snapshot. This is done without a further selfconsistent calculation of the non-collinear state, as such a calculation requires many self-consistent steps while adjusting the necessary constraining fields, leading to an increase of computational time by one to two orders of magnitude. The resulting absolute values of magnetic moments differ only marginally from their respective values at the ground state, confirming the dominance of the intra-atomic exchange interaction over the inter-atomic ones. In the following, we briefly outline our implementation of the calculation of transport properties within the non-collinear formalism.

In the spirit of multiple-scattering theory and the KKR-GF method, we apply the spin rotations on the site-dependent $t$-matrices that are then used to calculate the non-collinear Green function via the Dyson equation. Thus the $t$-matrices are calculated as spin-diagonal quantities in a local spin frame (indicated by $t^{\mu(\text { loc })}$ ) and they are transformed to the global frame where they are indicated by $t^{\mu(\mathrm{glob})} \underline{\underline{42}}$ The transformation matrix $\mathbf{U}^{\mu}$, corresponding to the standard spherical rotation angles $\theta^{\mu}$ and $\phi^{\mu}$ at the site $\mu$, given by

$$
\mathbf{U}^{\mu}=\left[\begin{array}{cc}
\cos \left(\theta^{\mu} / 2\right) \mathrm{e}^{-\frac{\mathrm{i}}{2} \phi^{\mu}} & -\sin \left(\theta^{\mu} / 2\right) \mathrm{e}^{-\frac{\mathrm{i}}{2} \phi^{\mu}} \\
\sin \left(\theta^{\mu} / 2\right) \mathrm{e}^{\frac{i}{2} \phi^{\mu}} & \cos \left(\theta^{\mu} / 2\right) \mathrm{e}^{\frac{i}{2} \phi^{\mu}}
\end{array}\right],
$$

is used to mix the spin-up and spin-down components of the local $t$-matrix at energy $\varepsilon$, resulting in a $2 \times 2$ matrix in spin space $(\sigma=\uparrow, \downarrow)$

$$
\mathbf{t}_{L L^{\prime}}^{\mu(\text { glob })}(\varepsilon)=\mathbf{U}^{\mu} \operatorname{diag}\left[t_{L L^{\prime}}^{\mu \uparrow(\text { loc })}(\varepsilon), t_{L L^{\prime}}^{\mu \downarrow \text { (loc })}(\varepsilon)\right]\left(\mathbf{U}^{\mu}\right)^{\dagger},
$$

where $L=(l, m)$ is the angular momentum quantum number and $t_{L L^{\prime}}^{\mu \sigma \text { (loc) }}(\varepsilon)$ is calculated from the spindependent potential $V^{\mu \sigma}$ as

$$
t_{L L^{\prime}}^{\mu \sigma(\mathrm{loc})}(\varepsilon)=\int \mathrm{d} \mathbf{r} j_{L}(\mathbf{r}, \varepsilon) V^{\mu \sigma}(\mathbf{r}) R_{L^{\prime}}^{\mu \sigma}(\mathbf{r}, \varepsilon),
$$

where $j_{L}$ and $R_{L}^{\mu \sigma}$ are the Bessel functions and standard radial solutions to the Kohn-Sham potential at site $\mu$, respectively. The structural Green function matrix $\mathcal{G}$ of the system with dimension $2 \times N_{\text {at }} \times\left(l_{\max }+1\right)^{2}$, with $N_{\text {at }}$ the number of atoms, is obtained via the Dyson equation

$$
\mathcal{G}(\varepsilon)=\mathcal{G}_{0}(\varepsilon)\left\{\mathbf{I}-\left[\mathbf{t}^{\text {(glob) }}(\varepsilon)-\mathbf{t}_{0}(\varepsilon)\right]^{-1} \mathcal{G}_{0}(\varepsilon)\right\}^{-1},
$$

where $\mathbf{I}$ is the unit matrix and $\mathcal{G}_{0}$ and $\mathbf{t}_{0}$ are the reference system Green function matrix and $t$-matrix, respectively. After a Fourier transform, taking the periodic supercell geometry into account, the Green function is calculated for each momentum channel $\mathbf{k}_{\|}$. The transmission probability matrix in spin space as a function of $\mathbf{k}_{\|}$and $\varepsilon$ is calculated as

$$
\begin{gathered}
\Gamma^{\sigma \sigma^{\prime}}(\varepsilon)=\sum_{\mu \mu^{\prime}} \sum_{L L^{\prime}} \sum_{L^{\prime \prime} L^{\prime \prime \prime}}\left(J_{L L^{\prime \prime}}^{\mu \sigma}-J_{L^{\prime \prime} L}^{\mu \sigma *}\right)\left(J_{L^{\prime} L^{\prime \prime \prime}}^{\mu^{\prime} \sigma^{\prime}}-J_{L^{\prime \prime \prime} L^{\prime}}^{\mu^{\prime} \sigma^{\prime}}\right) \\
\times \mathcal{G}_{L L^{\prime}}^{\mu \mu^{\prime} \sigma \sigma^{\prime}} \mathcal{G}_{L^{\prime \prime} L^{\prime \prime \prime}}^{\mu \mu^{\prime} \sigma}
\end{gathered}
$$

where $J_{L L^{\prime}}^{\mu \sigma}$ is the corresponding current-density matrix element in the non-magnetic lead in a cell associated with the site $\mu$ with the volume $\Omega_{\mu}$

$$
J_{L L^{\prime}}^{\mu \sigma}(\varepsilon)=\frac{1}{d_{\mathrm{at}}} \int_{\Omega_{\mu}} \mathrm{d} \mathbf{r} R_{L}^{\mu \sigma}(\mathbf{r}, \varepsilon) \partial_{z} R_{L^{\prime}}^{\mu \sigma}(\mathbf{r}, \varepsilon),
$$

and $d_{\mathrm{at}}$ is the atomic layer thickness $\stackrel{28}{2}$ The transmission probability matrix $\boldsymbol{\Gamma}(\varepsilon)$ is calculated between atomic pairs of the left and right lead, selected in a way that the whole cross-section of the lead is covered (see Fig. 1). As it was shown previously, using one atomic layer on each side yields well converged results in the fcc lattice system. 28 We verified it for the TL system by varying the number of atomic layers used for the transmission probability calculation. The difference between $\boldsymbol{\Gamma}$ calculated using one and two layers in the leads did not exceed $0.3 \%$.

While quantities within the KKR-GF approach are usually evaluated on a complex energy contour, the transport coefficients should be calculated from the transmission probability evaluated on the real energy axis. In the proximity of the real energy axis, a linear dependence of $\Gamma(\varepsilon)$ on $\Im(\varepsilon)$ is expected 22 Thus, the transmission probability $\boldsymbol{\Gamma}(\varepsilon)$ can be calculated for several small values of the $\varepsilon$ imaginary part [but setting $\Im(\varepsilon)$ large enough to ensure numerical stability in the Green function calculation] and an estimate of $\boldsymbol{\Gamma}(E)$ [where $E=\Re(\varepsilon)$ ] is then obtained as a linear extrapolation of $\boldsymbol{\Gamma}(\varepsilon)$ to $\Im(\varepsilon)=0$. We indeed found a very close to linear behavior of $\boldsymbol{\Gamma}(\varepsilon)$ on small values of $\Im(\varepsilon)$ and for all production runs, we calculated $\boldsymbol{\Gamma}$ for two $\Im(\varepsilon)$ values corresponding to a smearing temperature of $20 \mathrm{~K}$ and $10 \mathrm{~K}$ and extrapolated the results to $0 \mathrm{~K}$.

The transport coefficients $\mathbf{L}_{n}$ were evaluated by a numerical integration of $\boldsymbol{\Gamma}\left(\mathbf{k}_{\|}, E\right)$ over a set of discrete values of the momentum $\mathbf{k}_{\|}$and energy $E$ as

$$
\mathbf{L}_{n}=-\int \mathrm{d} E \frac{\partial f_{T}(E)}{\partial E}\left(E-E_{\mathrm{F}}\right)^{n} \int_{\mathrm{SBZ}} \mathrm{d} \mathbf{k}_{\|} \boldsymbol{\Gamma}\left(\mathbf{k}_{\|}, E\right) .
$$


Here, $f_{T}(E)=\left[\exp \left(\frac{E-E_{\mathrm{F}}}{k_{\mathrm{B}} T}\right)+1\right]^{-1}$ is the Fermi-Dirac distribution function with $T$ corresponding to the temperature of the MC simulation, $E_{\mathrm{F}}$ is the Fermi energy and $d \mathbf{k}_{\|}$is the integration element in the SBZ. For each temperature and system, $\boldsymbol{\Gamma}(E)=\int_{\mathrm{SBZ}} \mathrm{d} \mathbf{k}_{\|} \boldsymbol{\Gamma}\left(\mathbf{k}_{\|}, E\right)$ was calculated on an individual grid of 21 equidistant $E$ points in the range from $-10 k_{\mathrm{B}} T$ to $+10 k_{\mathrm{B}} T$ as the $\left(E-E_{\mathrm{F}}\right)^{n} \partial f_{T} / \partial E$ factors become negligible at $\pm 10 k_{\mathrm{B}} T$. We tested a four times denser $E$ grid for the TL system and found no significant change in the results. Finally we averaged over the non-collinear MC configurations at a given temperature obtaining $\left\langle\mathbf{L}_{n}\right\rangle_{T}$.

The transport quantities, namely, electrical conductance $G$, electrical resistance $R$, charge Seebeck coefficient $S_{\mathrm{C}}$ and spin Seebeck coefficient $S_{\mathrm{S}}$, are finally calculated using the well-known formulas

$$
\begin{aligned}
G^{\sigma \sigma^{\prime}}(T) & =\frac{e^{2}}{h}\left\langle L_{0}^{\sigma \sigma^{\prime}}\right\rangle_{T} \\
G & =\sum_{\sigma \sigma^{\prime}} G^{\sigma \sigma^{\prime}} \\
R & =\frac{1}{G} \\
S_{\mathrm{C}} & =-\frac{\sum_{\sigma \sigma^{\prime}}\left\langle L_{1}^{\sigma \sigma^{\prime}}\right\rangle_{T}}{e T \sum_{\sigma \sigma^{\prime}}\left\langle L_{0}^{\sigma \sigma^{\prime}}\right\rangle_{T}} \\
S_{\mathrm{S}} & =-\frac{\left\langle L_{1}^{\uparrow \uparrow}\right\rangle_{T}+\left\langle L_{1}^{\downarrow \uparrow}\right\rangle_{T}-\left\langle L_{1}^{\downarrow \downarrow}\right\rangle_{T}-\left\langle L_{1}^{\uparrow \downarrow}\right\rangle_{T}}{e T \sum_{\sigma \sigma^{\prime}}\left\langle L_{0}^{\sigma \sigma^{\prime}}\right\rangle_{T}} .
\end{aligned}
$$

\section{RESULTS}

\section{A. Electronic structure of the ground state}

Before we proceed to the analysis of the spin disorder effect on the transport properties, we want to point out the characteristic features of the studied systems, as well as their similarities and/or differences. For that purpose, the electron density of states (DOS) of selected systems is depicted in Fig. ए(a). For brevity, we refer to the majority and minority spin channel as $\uparrow$ and $\downarrow$, respectively and we use the abbreviations for the model systems as introduced in Fig. 2. For all systems in the ordered magnetic state, the $\operatorname{DOS}^{\uparrow}\left(E_{\mathrm{F}}\right)$ of Co atoms (open squares) as well as the $\operatorname{DOS}^{\uparrow / \downarrow}\left(E_{\mathrm{F}}\right)$ of $\mathrm{Cu}$ atoms (open circles) is rather low due to formally filled $d$ orbitals. In the TL system, partially occupied minority spin $d$ orbitals of Co atoms yields the $\operatorname{DOS}^{\downarrow}\left(E_{\mathrm{F}}\right)$ about three times larger than the $\operatorname{DOS}^{\uparrow}\left(E_{\mathrm{F}}\right)$ (not shown). The one-dimensional character of the nanowires is expected to manifest itself via Van Hove singularity features in the DOS. The DOS $\downarrow$ at around $E_{\mathrm{F}}$ is indeed raised for all nanowires, but a pronounced peak can be seen only in the W11(Va) system. We note that a strong asymmetry of the DOS around $E_{\mathrm{F}}$, as seen for $\mathrm{W} 11(\mathrm{Va})$, was suggested as an indicator of a large Seebeck coefficient 17

In Fig. 4(b), we show the transmission probability $\Gamma$ as a function of the energy $E$ around the Fermi level, (a)

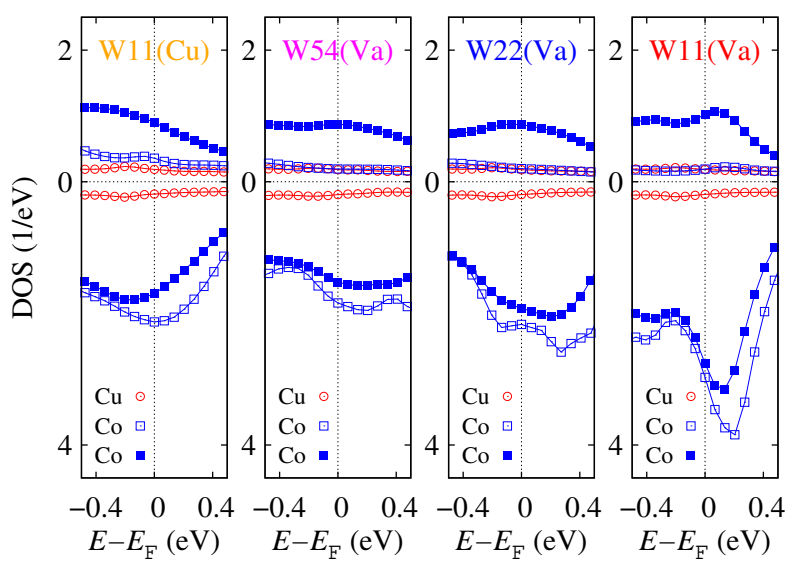

(b)

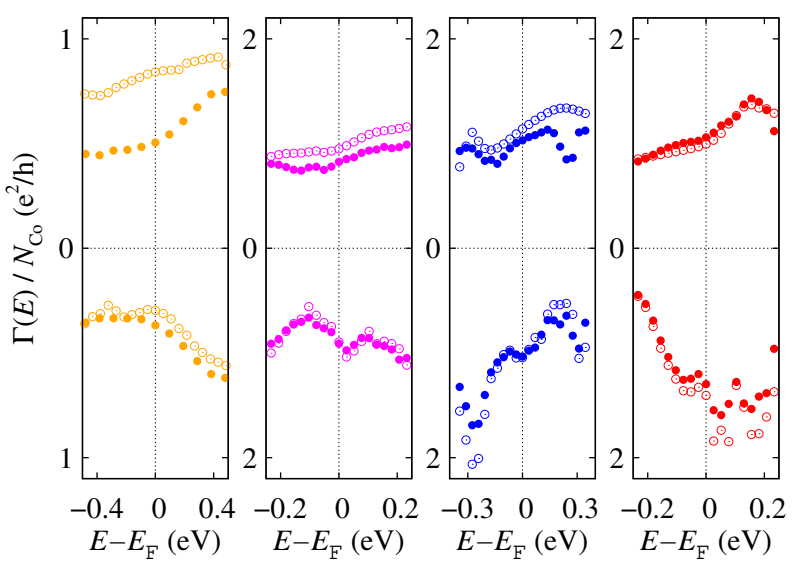

FIG. 4: (Color online) (a) The electron density of states (DOS) for majority and minority spin channel (upper and reverse scale lower part of the graphs, respectively) averaged over four $\mathrm{Cu}$ layers adjacent to the scattering region (circles) and all Co atoms in the system (squares). Data corresponding to the spin-ordered and spin-disordered case (calculated at MC simulation $T \approx 1100 \mathrm{~K}$ ) is shown as open and filled symbols, respectively. (b) Transmission probability $\Gamma$ divided by the number of Co atoms $\left(N_{\mathrm{Co}}\right)$ at the most narrow constriction of the respective system $\left(N_{\mathrm{Co}}\right.$ is $9,4,2,1$ for the TL, W54, W22, W11 systems, respectively), corresponding to the systems in (a). Open and filled symbols correspond to the spin-ordered and average spin-disordered case, respectively. In the spin-disordered case, upper and reverse scale lower part of the graphs depict $\Gamma^{\uparrow}=\Gamma^{\uparrow \uparrow}+\left(\Gamma^{\uparrow \downarrow}+\Gamma^{\downarrow \uparrow}\right) / 2$ and $\Gamma^{\downarrow}=\Gamma^{\downarrow \downarrow}+\left(\Gamma^{\uparrow \downarrow}+\Gamma^{\downarrow \uparrow}\right) / 2$, respectively. The MC simulation temperature of the spin-disordered data (left to right) corresponds to $1100,300,400$, and $300 \mathrm{~K}$. The color coding of data sets in (b) is consistent with the system labels in Fig. 2.

divided by the number of Co atoms $\left(N_{\mathrm{Co}}\right)$ at the most narrow constriction of the respective system $\left(N_{\mathrm{Co}}\right.$ is 9 , 4, 2, 1 for the TL, W54, W22, W11 systems, respectively). The transmission probability $\Gamma^{\uparrow}$ at $E_{\mathrm{F}}$ is in the spin-ordered state (open symbols) slightly smaller than 1 and a slowly growing function of $E$ consistently for all systems. The $\Gamma^{\downarrow}$ exhibits much richer variation of its character. For example, despite the similarity of the DOS between W54(Va) and W22(Va), the correspond- 


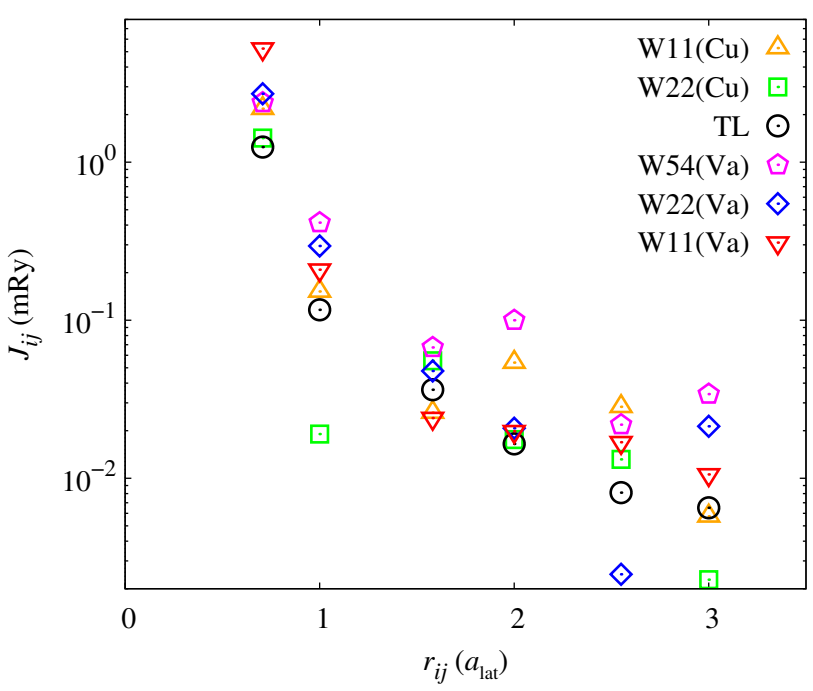

FIG. 5: (Color online) Absolute value of the leading exchange coupling parameters $J_{i j}$ in logarithmic scale as a function of the Co inter-site distance $r_{i j}$. The color coding of data sets is consistent with the system labels in Fig. 2.

ing $\Gamma^{\downarrow}$ as a function of $E$ is very different. A discussion of the spin disorder effect on the DOS and $\boldsymbol{\Gamma}$ will follow later, together with an analysis of individual transport properties.

\section{B. Spin disorder}

As outlined in the previous section, the spin disorder is determined by the fluctuations of the magnetic moments obtained from snapshots of MC simulations of the classical Heisenberg Hamiltonian. The exchange coupling parameters $J_{i j}$ between the Co atoms were calculated for all pairs for which the distance $r_{i j} \leq 3 a_{\text {lat }}$. We verified that a further increase of $r_{i j}$ did not affect results. The $J_{i j}$ parameters between the periodic images of the nanowires due to the in-plane periodicity were neglected, as their values were at least 3 orders of magnitude smaller than the nearest neighbor exchange coupling. Figure 5 shows the absolute value of the leading $J_{i j}$ terms in logarithmic scale, showing that the nearest and next-nearest neighbor interactions are dominant.

The magnetization and susceptibility as a function of the MC simulation temperature is shown in Fig. 6(a). Since there is no real critical temperature in nanostructures, we define $T_{\mathrm{c}}$ as a crossover temperature at the magnetic susceptibility peak. While the TL system exhibits critical-like behavior at around $1100 \mathrm{~K}$ (sharp drop of the magnetic moment and divergence character of the magnetic susceptibility), all nanowires retain a rather large magnetic moment until very high temperatures, resembling macro-spin character. The reduction of magnetic moment at high temperatures is less pronounced for the nanowires with smaller cross-section. The divergence character of their magnetic susceptibility, as a possible indication of the crossover temperature, is strongly suppressed and the corresponding peaks are shifted to much lower temperatures.

As we will show later, certain features of the spin disorder effects on the transport properties could not be correlated with the position of the susceptibility peak. Therefore, we explore an alternative sign of the magnetic order loss, the spatial correlation $C_{N}(T)$ as defined in Eq. (3). In Fig. 6(b), the $C_{N}(T)$ calculated between the moments of the $N$-th nearest neighbor layers in the $z$ direction, is shown in logarithmic scale for $N=\{1, \ldots, 7\}$. The nearest neighbor spatial correlation $C_{1}$ (thickest line) when seen in linear scale (not shown) has a very similar evolution with $T$ as the magnetic moment. The spatial correlation between farther neighbors of the different systems exhibits very diverse trends. In the case of TL, all farther-neighbor $C_{N \geq 2}$ terms tightly follow the $C_{1}$ up to $T_{\mathrm{c}}$. Above this temperature, the falloff of $C_{1}$ and $C_{2}$ is less steep than that of $C_{N>3}$. This is consistent with the presence of short-range magnetic order well above $T_{\mathrm{c}}$ while the long-range order is quickly suppressed. In the case of nanowires, the $C_{N}$ tend to quickly deviate from each other already at low temperatures. The nanowires embedded in $\mathrm{Cu}$ share a similar picture with the TL, exhibiting a fast decay of $C_{N \geq 3}$. The nanowires embedded in vacuum display a somewhat different trend, with no clear separation in the falloff of $C_{1}$ and $C_{2}$ with respect to $C_{N>3}$. Instructive is a comparison of the $\mathrm{W} 22(\mathrm{Cu})$ and $\mathrm{W} 11$ (Va) nanowires, for which the susceptibility peaks at around the same temperature. The $C_{1}$ and $C_{2}$, however, stay quite large at high $T$ in the case of W11(Va), suggesting well preserved short-range magnetic order and possibly different character of the spin disorder effect on the transport properties in comparison with $\mathrm{W} 22(\mathrm{Cu})$. Using the spatial correlation, we determine an independent measure of the long-range magnetic order loss. Especially for the TL, the crossover point $\left(T_{\mathrm{c}} \approx 1100 \mathrm{~K}\right)$ is very well approximated by using the condition that the $C_{3}$ value falls below 0.12 [indicated as dotted vertical and horizontal line in Fig. 6(a) and 6(b), respectively]. However, this is not the case for the nanowire systems, where the difference between the $T_{\mathrm{c}}$ and a crossover point determined from the falloff of $C_{3}$ is quite large. This suggests that the loss of order in the nanowires is more gradual, resulting in a strong drop of $C_{3}$ at higher temperatures than $T_{\mathrm{c}}$.

\section{Transport properties}

The electrical conductance of the spin-ordered and spin-disordered states as a function of temperature is shown in Fig. 6(c) and Fig. 6(d), respectively. As it can be seen in Fig. 6(c), the temperature dependence of the electrical conductance calculated using only the Fermi function smearing for the ordered magnetic configuration is very weak. Interestingly, the conductance 
(a)

(b)
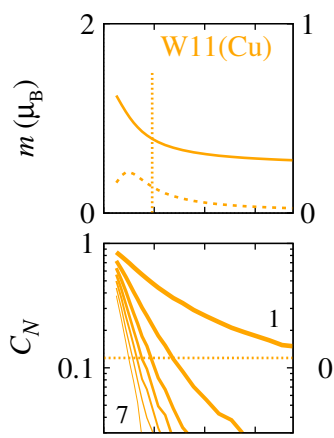

(c)

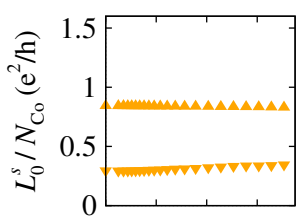

(d)

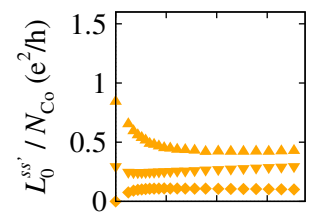

(e)

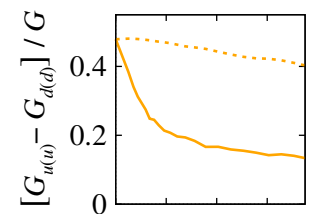

(f)

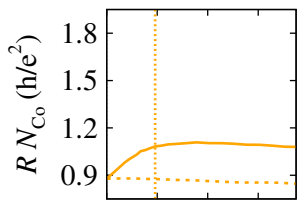

(g)

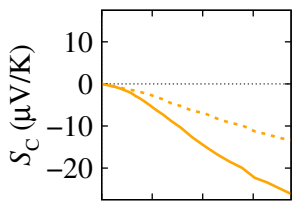

(h)

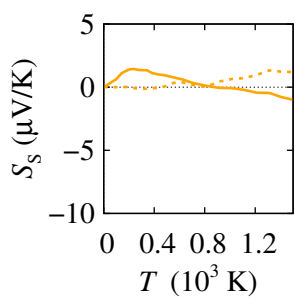

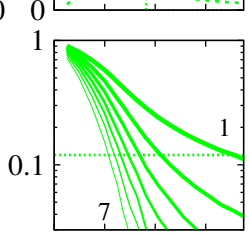
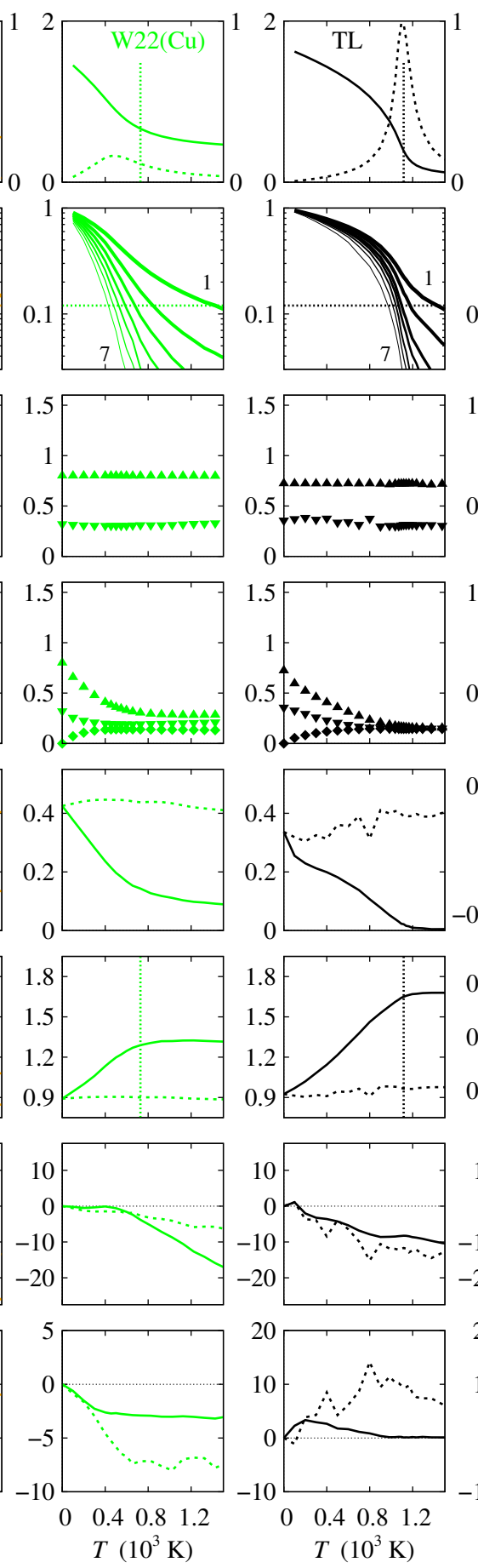
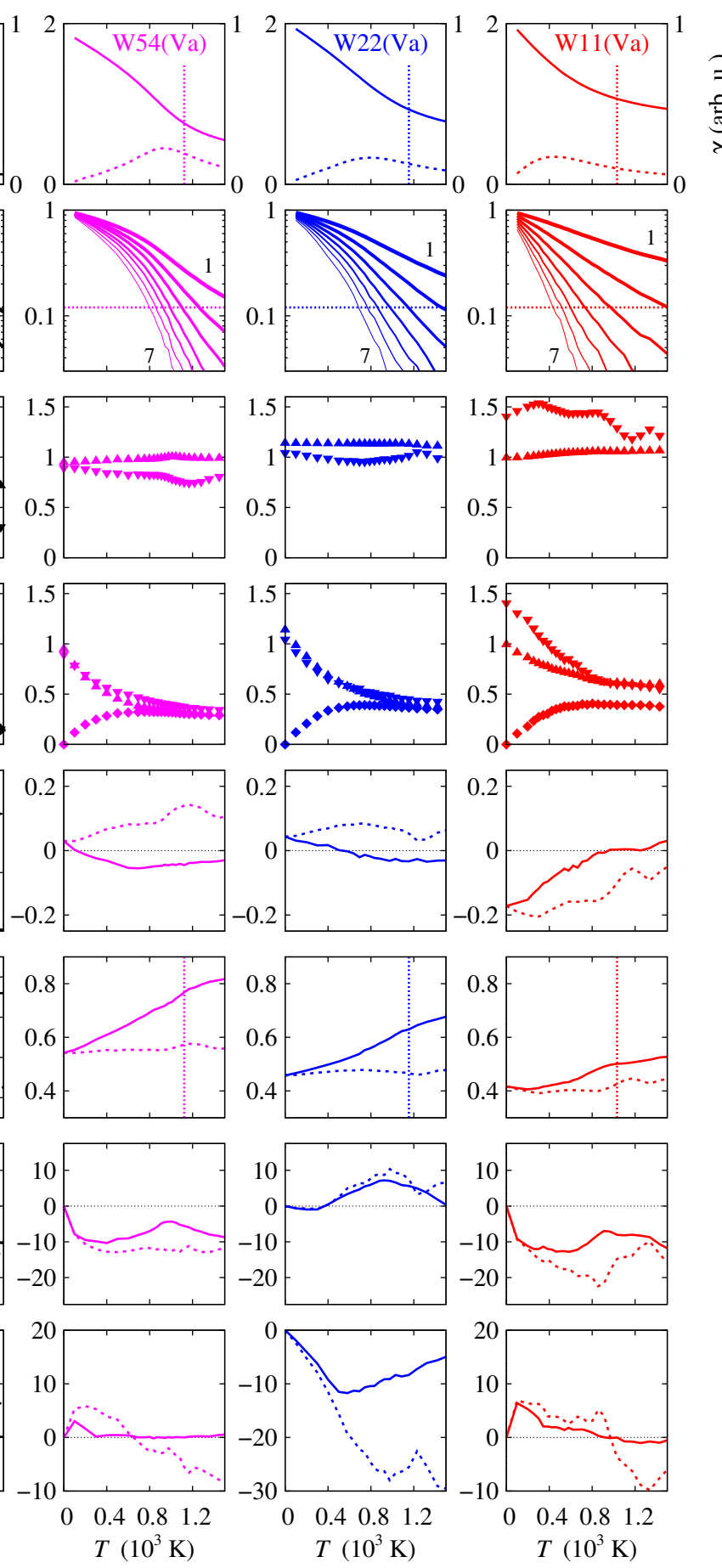

FIG. 6: (Color online) (a) Monte Carlo (MC) site averages of the Co atoms magnetic moment $m$ (solid line) and magnetic susceptibility $\chi$ (dashed line). (b) Spatial correlation $C_{N}$ of the magnetic moment orientation between $N$-th nearest neighbor layers in the $z$ direction. Thick-to-thin line corresponds to $1^{\text {st }}$ to $7^{\text {th }}$ nearest neighbors, respectively. Vertical dotted line in (a) indicates temperature for which $C_{3}$ drops under 0.12 [dotted line in (b)]. (c) Electrical conductance of the ordered magnetic configuration, the temperature dependence enters via Fermi function smearing. Triangles pointing up/down correspond to $\uparrow / \downarrow$ spin, respectively. (d) Electrical conductance of the spin-disordered magnetic configurations calculated by MC method. Triangles pointing up/down and diamonds correspond to $\uparrow \uparrow / \downarrow \downarrow$ and average of $\uparrow \downarrow$ and $\downarrow \uparrow$ of spin matrix elements, respectively. (e) Polarization of the electrical conductance. (f) Total electrical resistance. Position of the vertical dotted line is equivalent to (a). (g) Charge and (h) spin Seebeck coefficient. In (e-h), dashed and solid lines correspond to the spin-ordered and -disordered data, respectively. The color coding of data sets in the individual columns corresponds to the system labels in Fig. 2 . 
of both $\mathrm{W} 11(\mathrm{Cu})$ and $\mathrm{W} 22(\mathrm{Cu})$ is very similar to the TL system, although the number of Co atoms in the supercells containing a nanowire is much lower. On the contrary, the relative magnitude of the conductance via the majority and minority spin channels is reversing with decreasing thickness of the nanowires embedded in vacuum. As expected, the temperature effect on the electrical conductance via disordered magnetic configurations is significant [Fig. [6(d)]. The individual spin matrix elements of the conductance tend to converge at high temperatures. While the convergence is rather quick for the TL, it is suppressed as the cross-section of the nanowires decreases. This correlates with the fact that the $\uparrow$ and $\downarrow$ channels of the DOS and transmission probability for the nanowires are not equalized by the spin disorder even at $T \approx 1100 \mathrm{~K}$ [shown as filled symbols in Fig. 4(a) and 4(b) - only $\mathrm{W} 11(\mathrm{Cu})]$. The resulting electrical conductance polarization [Fig. 6(e)] shows that small variations in the geometry of nanowires can lead to large differences in the polarization [e.g., negative sign for W11(Va)]. Furthermore, the spin disorder has, in general, indeed a significant influence (solid line). Besides strong suppression of the polarization $[\mathrm{W} 11(\mathrm{Cu}), \mathrm{W} 22(\mathrm{Cu}), \mathrm{TL}]$, it can lead to sign reversal (nanowires embedded in vacuum).

The well-known effect of spin disorder resistivity can be seen in Fig. 6(f). A characteristic kink in the electrical resistance of the TL correlates with the crossover point observed in the MC data. This kink is present also in the nanowires embedded in copper (although it is not as sharp) and the resistance saturates above a certain temperature. The position of the kink (indicated by a vertical dotted line) correlates rather with the loss of long-range magnetic order $\left(C_{N \geq 3} \lesssim 0.12\right)$ than with the peak in magnetic susceptibility $\chi$. The kink is virtually missing in the nanowires embedded in vacuum where the resistance grows throughout the whole considered temperature range. Yet, a small change of slope in $R(T)$ can be identified, which again correlates rather with the long-range magnetic order loss than with the susceptibility peak.

While features of electrical conductance can be, for some simple systems, related to the electronic density of states, it is next to impossible to find such relation in case of the Seebeck coefficient, except perhaps in the lowtemperature limit of some model systems. The reason is that the Seebeck coefficient is a product of two transport coefficients $1 / L_{0}$ and $L_{1}$, where the former may already have not much relation to the DOS and the latter is resulting from contributions of the transmission probability $\Gamma(E)$, with maximum weight at $\left|E-E_{\mathrm{F}}\right| \approx 1.5 k_{\mathrm{B}} T$ and significant weight up to as far as $\left|E-E_{\mathrm{F}}\right| \approx 5 k_{\mathrm{B}} T$.

In Fig. 6(g) and Fig. 6(h), we present the results of the conventional charge $\left(S_{\mathrm{C}}\right)$ and spin $\left(S_{\mathrm{S}}\right)$ Seebeck coefficient, respectively. The influence of temperature is obviously non-trivial, even if only the effect due to the Fermi function smearing for the ordered spin configuration is considered (dashed line). Looking first at $S_{\mathrm{C}}$, a very fast onset can be seen for W54(Va) and W11(Va), quickly reaching almost its maximum value already at around or even well below room temperature. This result could be interpreted in the light of the hypothesis that a large Seebeck coefficient can be predicted from a steep $\operatorname{DOS}(E)$ slope at $E_{\mathrm{F}}$ due to Van Hove singularity in quantum wires. While this is consistent for the W11(Va) nanowire, the DOS of the W54(Va) nanowire is very flat at around $E_{\mathrm{F}}$. The explanation lies in the character of the transmission probability [Fig. 4(b)]. For both systems and both spin channels the slope of $\Gamma(E)$ at around $E_{\mathrm{F}}$ is positive and relatively large leading to a fast growth of $S_{\mathrm{C}}(T)$. The resulting difference of the $\uparrow$ and $\downarrow$ slope of $\Gamma\left(E_{\mathrm{F}}\right)$ yields somewhat smaller $S_{\mathrm{S}}$. A complementary argument showing the inability of the DOS to reflect the Seebeck coefficient comes from a comparison of the W54(Va) and W22(Va) nanowires. Despite a close similarity in the DOS [Fig. 4(a)], the effective slope of $\Gamma^{\downarrow}(E)$ at around $E_{\mathrm{F}}$ is opposite in sign for these two systems [Fig. 4(b)], which leads to a negligible $S_{\mathrm{C}}$ and an enhanced $S_{\mathrm{S}}$ at room temperature for the W22(Va) nanowire [Fig. [6(g-h)]. The growth of $S_{\mathrm{S}}(T)$ slows down at higher $T$ due to sharp kinks in $\Gamma^{\downarrow}$ at approximately $-0.3 \mathrm{eV}$ and $0.2 \mathrm{eV}$ [Fig. [(b)] while the slope difference of $\Gamma^{\uparrow}$ and $\Gamma^{\downarrow}$ at elevated temperatures leads to a sign reversal of $S_{\mathrm{C}}$ in comparison with all other model systems.

The effect of spin disorder on the Seebeck coefficients [solid line in Fig. 6(g-h)] is again generally quite pronounced as it was in the case of the electrical conductance, its polarization and the electrical resistance. An interesting observation is the rather strong enhancement of $S_{\mathrm{C}}$ due to the spin disorder for the nanowires embedded in copper. The origin is obvious when looking at the transmission probability of the $\mathrm{W} 11(\mathrm{Cu})$ nanowire in Fig. 4 (b). The spin disorder (shown at $\approx 1100 \mathrm{~K}$ ) causes a drop of the majority spin $L_{0}$ coefficient while the kink at $\Gamma^{\uparrow}$ very close to $E_{\mathrm{F}}$ leads to a rise in the majority spin $L_{1}$ coefficient. The resulting $L_{1} / L_{0}$ ratio is then significantly enhanced. Very similar behavior is seen for the W22(Cu) nanowire, where the $S_{\mathrm{C}}$ enhancement due to the spin disorder is shifted to higher temperatures. This is caused by the already mentioned kink at $\Gamma^{\uparrow}$ positioned slightly away from $E_{\mathrm{F}}$ (not shown). Furthermore, the spin disorder is expected to significantly suppress the spin Seebeck coefficient which is, in general, indeed observed. However, for the $\mathrm{W} 22(\mathrm{Cu})$ and $\mathrm{W} 22(\mathrm{Va})$ nanowires, the $S_{\mathrm{S}}$ remains relatively large even at very high temperatures due to the non-vanishing difference of the $L_{1}$ transport coefficient for the majority and minority spin channels.

\section{SUMMARY}

We investigated the effect of temperature induced spindisorder on the transport through several Co nanostructures embedded between $\mathrm{Cu}$ leads. The calculation of the transport properties confirmed that, at elevated temperatures, spin disorder affects the value of the transport coefficients both qualitatively and quantitatively, and there- 
fore cannot be neglected in a theoretical analysis. Additionally, we find that there is no clear connection between the transport properties and the density of states, due to the complex convolution of the Fermi function derivative and the energy dependent transport coefficients. These conclusions apply to the resistance, charge and spin Seebeck coefficients.

The well-known spin-disorder contribution to the resistance is found to be significant in the systems we studied. The temperature, at which the characteristic kink in the resistance is observed, can be related to the onset of the long-range magnetic order loss, determined from the spatial correlation of the fluctuating local magnetic moments. We find a non-trivial behavior of the charge and spin Seebeck coefficient as a function of temperature, that does not follow a clear universal semi-quantitative or even qualitative rule, as a number of effects are factored in for its calculation, including the fluctuations of local magnetic moments, their temperature-dependent correlation, the quantum confinement due to the nanostructure geometry, the participating conducting states due to the Fermi distribution, and the interface transmission. Furthermore, we showed that a decrease, an enhancement or even a change of sign of the charge and spin Seebeck co- efficients can result from an interplay of the spin-disorder and the geometry in a particular microscopic structure.

It is obvious that the spin disorder constitutes only one of many effects that contribute to the spin-caloric transport at high temperatures, others being phonons or magnon-assisted spin transport, not considered in this work. However, our results show that spin-disorder at high temperatures cannot be neglected for a quantitative or even qualitative description of thermoelectric and spin-caloric coefficients in magnetic nanostructures.

\section{Acknowledgments}

Support from the Deutsche Forschungsgemeinschaft (SPP 1538 "Spin Caloric Transport") is gratefully acknowledged. Computational resources were provided by the groups of M. Ležaić, S. Lounis and Y. Mokrousov at the PGI-1 at Forschungszentrum Jülich as well as from the JARA-HPC from RWTH Aachen University under project jara0051. We are grateful to D. Comtesse, P. Entel, P. Kratzer, V. Popescu, C. E. Quiroga and L. Szunyogh for numerous enlightening discussions.
* Electronic address: r.kovacik@fz-juelich.de

1 K. Uchida, S. Takahashi, K. Harii, J. Ieda, W. Koshibae, K. Ando, S. Maekawa, and E. Saitoh, Nature 455, 778 (2008).

${ }^{2}$ H.-F. Lü, L.-C. Zhu, X.-T. Zu, and H.-W. Zhang, Appl. Phys. Lett. 96, 123111 (2010).

3 A. A. Tulapurkar and Y. Suzuki, Solid State Commun. 150, 466 (2010).

4 C. M. Jaworski, J. Yang, S. Mack, D. D. Awschalom, J. P. Heremans, and R. C. Myers, Nature Mater. 9, 898 (2010).

5 A. Slachter, F. L. Bakker, J.-P. Adam, and B. J. van Wees, Nature Phys. 6, 879 (2010).

6 H. Adachi, K.-i. Uchida, E. Saitoh, J.-i. Ohe, S. Takahashi, and S. Maekawa, Appl. Phys. Lett. 97, 252506 (2010).

7 H. Adachi, J.-i. Ohe, S. Takahashi, and S. Maekawa, Phys. Rev. B 83, 094410 (2011).

8 C. M. Jaworski, J. Yang, S. Mack, D. D. Awschalom, R. C. Myers, and J. P. Heremans, Phys. Rev. Lett. 106, 186601 (2011).

9 J. Flipse, F. L. Bakker, A. Slachter, F. K. Dejene, and B. J. van Wees, Nature Nanotech. 7, 166 (2012).

10 F. K. Dejene, J. Flipse, and B. J. van Wees, Phys. Rev. B 86, 024436 (2012).

11 G. E. W. Bauer, E. Saitoh, and B. J. van Wees, Nature Mater. 11, 391 (2012).

12 K.-i. Uchida, A. Kirihara, M. Ishida, R. Takahashi, and E. Saitoh, Jpn. J. Appl. Phys. 50, 120211 (2011).

13 M. Weiler, M. Althammer, F. D. Czeschka, H. Huebl, M. S. Wagner, M. Opel, I.-M. Imort, G. Reiss, A. Thomas, R. Gross, et al., Phys. Rev. Lett. 108, 106602 (2012).

14 A. Fukushima, K. Yagami, A. A. Tulapurkar, Y. Suzuki, H. Kubota, A. Yamamoto, and S. Yuasa, Jpn. J. Appl. Phys. 44, L12 (2005).
15 H. Katayama-Yoshida, T. Fukushima, V. A. Dinh, and K. Sato, Jpn. J. Appl. Phys. 46, L777 (2007).

16 A. Sugihara, M. Kodzuka, K. Yakushiji, H. Kubota, S. Yuasa, A. Yamamoto, K. Ando, K. Takanashi, T. Ohkubo, K. Hono, et al., Appl. Phys. Express 3, 065204 (2010).

17 N. D. Vu, K. Sato, and H. Katayama-Yoshida, Appl. Phys. Express 4, 015203 (2011).

18 V. Popescu and P. Kratzer, Phys. Rev. B 88, 104425 (2013).

19 S. Arajs and R. V. Colvin, Phys. Status Solidi 6, 797 (1964).

20 W. Kierspe, R. Kohlhaas, and H. Gonska, Z. Angew. Phys. 24, 28 (1967).

21 A. L. Wysocki, K. D. Belashchenko, J. P. Velev, and M. van Schilfgaarde, J. Appl. Phys. 101, 09G506 (2007).

22 Á. Buruzs, L. Szunyogh, and P. Weinberger, Philos. Mag. 88, 2615 (2008).

23 A. L. Wysocki, R. F. Sabirianov, M. van Schilfgaarde, and K. D. Belashchenko, Phys. Rev. B 80, 224423 (2009).

24 J. K. Glasbrenner, K. D. Belashchenko, J. Kudrnovský, V. Drchal, S. Khmelevskyi, and I. Turek, Phys. Rev. B 85, 214405 (2012).

25 J. Kudrnovský, V. Drchal, I. Turek, S. Khmelevskyi, J. K. Glasbrenner, and K. D. Belashchenko, Phys. Rev. B 86, 144423 (2012).

26 N. Papanikolaou, R. Zeller, and P. H. Dederichs, J. Phys.: Condens. Matter 14, 2799 (2002).

27 A. I. Liechtenstein, M. I. Katsnelson, V. P. Antropov, and V. A. Gubanov, J. Magn. Magn. Mater. 67, 65 (1987).

28 P. Mavropoulos, N. Papanikolaou, and P. H. Dederichs, Phys. Rev. B 69, 125104 (2004).

29 B. Y. Yavorsky and I. Mertig, Phys. Rev. B 74, 174402 
(2006).

30 N. Stefanou, H. Akai, and R. Zeller, Comput. Phys. Commun. 60, 231 (1990).

31 N. Stefanou and R. Zeller, J. Phys.: Condens. Matter 3, 7599 (1991).

${ }^{32}$ H. Ebert and R. Zeller, The SPR-TB-KKR package, URL: olymp.cup.uni-muenchen.de/ak/ebert/SPR-TB-KKR, also see kkr-gf.org.

33 S. H. Vosko, L. Wilk, and M. Nusair, Can. J. Phys. 58, 1200 (1980).

${ }^{34}$ F. Garcia-Moliner and V. R. Velasco, Prog. Surf. Sci. 21, 93 (1986).

35 L. Szunyogh, B. Újfalussy, P. Weinberger, and J. Kollár, Phys. Rev. B 49, 2721 (1994).
36 K. Momma and F. Izumi, J. Appl. Cryst. 44, 1272 (2011).

37 D. Bauer, PhD Thesis, RWTH Aachen University (2013).

38 N. Metropolis, A. W. Rosenbluth, M. N. Rosenbluth, A. H. Teller, and E. Teller, J. Chem. Phys. 21, 1087 (1953).

39 M. Matsumoto and T. Nishimura, ACM Trans. Model. Comput. Simul. 8, 3 (1998).

${ }^{40}$ V. P. Antropov, M. I. Katsnelson, B. N. Harmon, M. van Schilfgaarde, and D. Kusnezov, Phys. Rev. B 54, 1019 (1996).

41 S. V. Halilov, H. Eschrig, A. Y. Perlov, and P. M. Oppeneer, Phys. Rev. B 58, 293 (1998).

42 S. Lounis, P. Mavropoulos, P. H. Dederichs, and S. Blügel, Phys. Rev. B 72, 224437 (2005). 